\title{
THE ISOPERIMETRIC INEQUALITY AND ITS STABILITY
}

\author{
CHANG-JUn Li AND XIANG GAO
}

\begin{abstract}
In this paper, we deals with the isoperimetric-type inequalities for the closed convex curve in the Euclidean plane $\mathbb{R}^{2}$. In fact we establish a family of parametric inequalities involving the following geometric functionals associated to the given closed convex curve with a simple Fourier series proof: length of the curve, areas of the region included by the curve and the locus of curvature centers, and integral of the curvature radii of the curve and the locus of curvature centers. Using our isoperimetric-type inequalities, we also derive some new geometric Bonnesen-type inequalities. Furthermore, we investigate the stability property of such inequalities (near equality implies curve nearly circular).
\end{abstract}

Mathematics subject classification (2010): Primary 52A38; Secondary 52A40. Keywords and phrases: Isoperimetric inequality, Fourier series, stability.

\section{REFERENCES}

[1] J. STEINER, Sur le maximum et le minimum des figures dans le plan, sur la sphère, et dans l'espace en général, I and II, J. Reine Angew. Math. (Crelle), 24 (1842), 93-152 and 189-250.

[2] S. L. PAN, H. ZHANG, A reverse isoperimetric inequality for convex plane curves, Beitrage Algebra Geom. 48 (2007) 303-308.

[3] X. GAO, A note on the reverse isoperimetric inequality, Results. Math. 59 (2011), 83-90.

[4] S. L. PAN, J. N. YANG, On a non-local perimeter-preserving curve evolution problem for convex plane curves, Manuscripta Math. 127, (2008), 469-484.

[5] R. SchneIder, Convex bodies: The Brunn-Minkowski theory, Encyclopedia of Mathematics and its Applications, 44, Cambridge University Press, Cambridge, 1993.

[6] S. L. PAn, H. P. XU, Stability of a reverse isoperimetric inequality, J. Math. Anal. Appl. 350 (2009), 348-353.

[7] H. Groemer, Geometric applications of Fourier series and spherical harmonics, Encyclopedia of Mathematics and its Applications, 61, Cambridge University Press, Cambridge, 1996. 